\title{
A new modification of He's homotopy perturbation method for rapid convergence of nonlinear undamped oscillators
}

\author{
D.D. Ganji • A.R. Sahouli • M. Famouri
}

Received: 3 August 2008 / Revised: 13 September 2008 / Published online: 8 October 2008

(C) The Author(s) 2008. This article is published with open access at Springerlink.com

\begin{abstract}
In this paper we present a new efficient modification of the homotopy perturbation method with $x^{3}$ force nonlinear undamped oscillators for the first time that will accurate and facilitate the calculations. The He's homotopy perturbation method is modified by adding a term to linear operator depends on the equation and boundary conditions. We find that this modified homotopy perturbation method works very well for the wide range of time and boundary conditions for nonlinear oscillator. Only two or three iteration leads to high accuracy of the solutions. We then conduct a comparative study between the new modification and the homotopy perturbation method for strongly nonlinear oscillators. Numerical illustrations are investigated to show the accurate of the techniques. The new modified method accelerates the rapid convergence of the solution, reduces the error solution and increases the validity range. The new modification introduces a promising tool for many nonlinear problems.
\end{abstract}

Keywords Homotopy Perturbation Method (HPM) · Nonlinear undamped oscillator · Convergence

Mathematics Subject Classification (2000) $34 \mathrm{~L} 30 \cdot 55 \mathrm{P} 42 \cdot 37 \mathrm{C} 75$

\section{Introduction}

The development of numerical techniques for solving nonlinear algebraic equations is a subject of considerable interest. There are many papers that deal with nonlinear algebraic equations. The application of homotopy perturbation method in linear and nonlinear problems has been devoted by scientists and engineers [1-19], because this method is to continuously deform a simple problem which is easy to solve into the

D.D. Ganji ( $\varangle)$ · A.R. Sahouli · M. Famouri

Department of Mechanical Engineering, Babol University of Technology, P.O. Box 484, Babol, Iran e-mail: ddg_davood@yahoo.com 
under study problem which is difficult to solve. This method, homotopy perturbation method (HPM), proposed first by He [1, 2], for solving differential and integral equations, linear and nonlinear has been the subject of extensive analytical and numerical studies. The method is a coupling of the traditional perturbation method and homotopy in topology. This method, which does not require a small parameter in an equation, has a significant advantage in that it provides an analytical approximate solution to a wide range of nonlinear problems in applied sciences. This HPM has already been applied successfully to solve Laplace equation [3], nonlinear dispersive $K(\mathrm{mp})$ equations [4], heat radiation equations [5], nonlinear integral equations [6], nonlinear heat conduction and convection equations [7], nonlinear oscillators [8], nonlinear Schrödinger equations [9], nonlinear wave equations [10], nonlinear chemistry problems [11], and to other fields [11-19]. This HPM yields a very rapid convergence of the solution series in most cases, usually only a few iterations leading to very accurate solutions. Thus He's HPM is a universal one which can solve various kinds of nonlinear equations.

Recently, some modifications of this method have published to facilitate and accurate the calculations and accelerate the rapid convergence of the series solution and reduce the size of work [20-25] and some new methods were found to overcome the shortcomings, such as parameter-expansion method [36-41]. It is the purpose of the present paper to introduce a new reliable modification of the HPM. The new modification demonstrates an accurate solution if compared with standard HPM, and therefore it has been shown that to be computationally efficient in applied fields. In addition the new modified HPM may give the exact solution for nonlinear equations by using two iterations only. The obtained results suggest that this newly improvement technique introduces a powerful improvement for solving nonlinear problems.

In this study, we have applied new modification HPM to find the approximate solutions of nonlinear differential equation governing strongly nonlinear oscillators and have made a comparison with the numerical solution. MATLAB software [26] command ODE45 has been used and considered as numerical solution.

\section{Analysis of the method}

\subsection{The standard HPM}

To illustrate the basic ideas of this method, we consider the following nonlinear differential equation:

$$
A(u)-f(r)=0, \quad r \in \Omega
$$

Considering the boundary conditions of:

$$
B(u, \partial u / \partial n)=0, \quad r \in \Gamma,
$$

where $A$ is a general differential operator, $B$ a boundary operator, $f(r)$ a known analytical function and $\Gamma$ is the boundary of the domain $\Omega$. 
The operator $A$ can be divided into two parts of $L$ and $N$, where $L$ is the linear part, while $N$ is a nonlinear one. Equation (2.1) can, therefore, be rewritten as:

$$
L(u)+N(u)-f(r)=0
$$

By the homotopy technique, we construct a homotopy as $v(r, p): \Omega \times[0,1] \rightarrow \Re$ which satisfies:

$$
H(v, p)=(1-p)\left[L(v)-L\left(u_{0}\right)\right]+p[A(v)-f(r)]=0, \quad p \in[0,1], r \in \Omega,
$$

Where $p \in[0,1]$, is an embedding parameter and $u_{0}$ is an initial approximation which satisfies the boundary conditions. Obviously, considering (2.4) we will have:

$$
\begin{aligned}
& H(v, 0)=L(v)-L\left(u_{0}\right)=0 \\
& H(v, 1)=A(v)-f(r)=0
\end{aligned}
$$

The changing process of $p$ from zero to unity is just that of $v(r, p)$ from $u_{0}(r)$ to $u(r)$. In topology, this is called deformation, and $L(v)-L\left(u_{0}\right)$ and $A(v)-f(r)$ are called homotopy. According to HPM, we can first use the embedding parameter $p$ as "small parameter", and assume that the solution of (2.4) can be written as a power series in $p$ :

$$
v=v_{0}+p v_{1}+p^{2} v_{2}+\ldots
$$

Setting $p=1$ result in the approximate solution of (2.4):

$$
u=\lim _{p \rightarrow 1} v=v_{0}+v_{1}+v_{2}+\ldots
$$

The combination of the perturbation method and the homotopy method is called the HPM, which lacks the limitations of the traditional perturbation methods, although this technique can have full advantages of the traditional perturbation techniques.

The series (2.7) is convergent for most cases. However, the convergence rate depends on the nonlinear operator $A(v)$. The following opinions are suggested by He $[1,2]$ :

1. The second derivative of $N(v)$ with respect to $v$ must be small because the parameter $p$ may be relatively large, i.e. $p \rightarrow 1$.

2. The norm of $L^{-1} \partial N / \partial v$ must be smaller than one so that the series converges.

\subsection{The new modified HPM}

The present new modified HPM that is used to solve the nonlinear undamped oscillator is similar to standard HPM. In this way, we add and subtract a linear term such as $C x(t)$ to the main governing equation which $x$ is depending variable, $t$ is independent variable and $C$ is a constant coefficient. In this way the governing equation remain unchanged, but we choose just one of the additional terms for using in linear operator. This term which is added to liner operator, prevent from divergent solution and it cusses the rapid convergent and much accurate solution if compared with the 
standard HPM. The only different between present HPM and standard HPM is the linear operator and the other procedures are the same for both cases.

The additional term depends on the form of the main equation and boundary conditions that this term is changed from a problem to another one. The presented modified linear operator plays the main rule in solution. This new modified of homotopy perturbation method is introduced for the first time and it can be developed for many oscillator and none oscillator problems which standard HPM can't solve them as well.

\section{Applications}

To demonstrate the effectiveness of the proposed new modification and to compare the new modification of the HPM with the standard HPM, we have chosen differential equations as:

$$
\ddot{x}+\alpha x+\varepsilon x^{3}=0
$$

Subjected to initial condition as:

$$
x(0)=A, \quad \dot{x}(0)=0
$$

There are several studies on this nonlinear equation with different value of $\alpha$ and $\varepsilon$ [27-35]. Various types of this equation studied by means of Adomian decomposition [27], artificial parameter-decomposition [28], variational iteration method [29, 34, 35], He's parameter-expanding [30] and homotopy perturbation method [32].

\subsection{The standard HPM}

In view of the homotopy (2.4), and after separating the linear and nonlinear parts, we have:

$$
(1-p)\left(\ddot{x}+\alpha x-\left(\ddot{x}_{0}+\alpha x_{0}\right)\right)+p\left(\ddot{x}+\alpha x+\varepsilon x^{3}\right)=0
$$

Assume that the solution of (3.3) can be written as a power series in $p$ :

$$
x=x_{0}+p x_{1}+p^{2} x_{2}+\ldots
$$

Substituting (3.3a) and the initial conditions (3.2) into the homotopy (3.3) and equating the terms with identical powers of $p$, we obtain the following set of linear differential equations:

$$
\begin{array}{ll}
p^{0}: \ddot{x}_{0}+\alpha x_{0}=0, & x_{0}(0)=A, \dot{x}_{0}(0)=0 \\
p^{1}: \ddot{x}_{1}+\alpha x_{1}+\varepsilon x_{0}^{3}=0, & x_{1}(0)=0, \dot{x}_{1}(0)=0 \\
p^{2}: \ddot{x}_{2}+\alpha x_{2}+3 \varepsilon x_{0}^{2} x_{1}=0, & x_{2}(0)=0, \dot{x}_{2}(0)=0 \\
p^{3}: \ddot{x}_{3}+\alpha x_{3}+3 \varepsilon x_{0}^{2} x_{2}+3 \varepsilon x_{0} x_{1}^{2}=0, & x_{3}(0)=0, \dot{x}_{3}(0)=0 \\
p^{4}: \ddot{x}_{4}+\alpha x_{4}+3 \varepsilon x_{0}^{2} x_{3}+6 \varepsilon x_{0} x_{1} x_{2}+\varepsilon x_{1}^{3}=0, & x_{4}(0)=0, \dot{x}_{4}(0)=0
\end{array}
$$


Consequently, solving the above equations, the first few components of the homotopy perturbation solution for (3.4) are derived as follows when $\alpha \neq 0$ :

$$
\begin{aligned}
x_{0}= & A \cos (\sqrt{\alpha} t) \\
x_{1}= & \frac{-\varepsilon A^{3}}{32 \alpha^{\frac{3}{2}}}(\cos (\sqrt{\alpha} t) \sqrt{\alpha}-\cos (3 \sqrt{\alpha} t) \sqrt{\alpha}+12 t \sin (\sqrt{\alpha} t) \alpha) \\
x_{2}= & \frac{\varepsilon^{2} A^{5}}{1024 \alpha^{\frac{5}{2}}}(23 \cos (\sqrt{\alpha} t) \sqrt{\alpha}-36 t \sin (3 \sqrt{\alpha} t) \alpha+\cos (5 \sqrt{\alpha} t) \sqrt{\alpha} \\
& -24 \cos (3 \sqrt{\alpha} t) \sqrt{\alpha} \\
& \left.+96 t \sin (\sqrt{\alpha} t) \alpha-72 \cos (\sqrt{\alpha} t) a^{\frac{3}{2}} t^{2}\right)
\end{aligned}
$$

When $\alpha$ reads zero, solving the above equations results in the following answers:

$$
\begin{array}{ll}
x_{0}=A, & x_{1}=-\frac{\varepsilon A^{3} t^{2}}{2}, \\
x_{2}=\frac{\varepsilon^{2} A^{5} t^{4}}{8}, & x_{3}=-\frac{3 \varepsilon^{3} A^{7} t^{6}}{80},
\end{array}
$$

Having $x_{i}, i=1,2, \ldots, n$, the solutions are as follows:

$$
x(t)=x_{0}(t)+x_{1}(t)+\ldots+x_{n}(t)
$$

\subsection{The new modified HPM}

To illustrate the new modified HPM or rather new modified linear operator, we consider the additional term as $f(A)$ for (3.1) which $A$ comes from the initial condition; this manner let us to choose a additional term function of boundary conditions; in this way we have:

$$
\ddot{x}+f(A) x-f(A) x+\alpha x+\varepsilon x^{3}=0
$$

Now, i.e., we consider just one of the additional terms for linear operator:

$$
L(x): \equiv \ddot{x}+f(A) x+\alpha x
$$

If we consider the solution as $x=\sum_{k=0}^{n} \beta_{k} e^{s_{k} t}$ and substitute to the characteristic equation of governing equation, we have:

$$
s_{k}^{2}\left(\sum_{k=0}^{n} \beta_{k} e^{s_{k} t}\right)+\alpha\left(\sum_{k=0}^{n} \beta_{k} e^{s_{k} t}\right)+\varepsilon\left(\sum_{k=0}^{n} \beta_{k} e^{s_{k} t}\right)^{3}=0
$$


With considering $x=\left(\sum_{k=0}^{n} \beta_{k} e^{s_{k} t}\right) \neq 0$, it simplifies to:

$$
s_{k}^{2}=-\left(\alpha+\varepsilon\left(\sum_{k=0}^{n} \beta_{k} e^{s_{k} t}\right)^{2}\right)
$$

On the other hand, if we expand (2.4) with (3.7), the coefficient of $P^{0}$ is:

$$
\ddot{x}_{0}+f(A) x_{0}+\alpha x_{0}=0
$$

Characteristic equation of this equation is written as:

$$
s_{0}^{2}=-f(A)-\alpha
$$

From (3.11), we have

$$
\begin{aligned}
& s_{0}^{2}=-\left(\alpha+\varepsilon\left(\beta_{0} e^{s_{0} t}\right)^{2}\right) \\
& x_{0}=\beta_{0} e^{s_{0} t}=A e^{s_{0} t}
\end{aligned}
$$

From (3.14), we have

$$
s_{0}^{2}=-\left(\alpha+\varepsilon\left(A e^{s_{0} t}\right)^{2}\right)
$$

From (3.13) and (3.15), we have:

$$
f(A)=\varepsilon\left(A e^{s_{0} t}\right)^{2}
$$

Because $f(A)$ is an integral number, thus we have:

$$
\begin{aligned}
& |f(A)|=\left|\varepsilon\left(A e^{s_{0} t}\right)^{2}\right| \\
& |f(A)|=\left|\varepsilon\left(A e^{s_{0} t}\right)^{2}\right|=\varepsilon A^{2} \\
& f(A)=\varepsilon A^{2}
\end{aligned}
$$

In view of the (3.7) and (3.16), we have:

$$
\ddot{x}+\left(\varepsilon A^{2}+\alpha\right) x-\varepsilon A^{2} x+\varepsilon x^{3}=0
$$

We construct the following homotopy and consider the linear term as $\ddot{x}+\left(\varepsilon A^{2}+\alpha\right) x$ :

$$
(1-p)\left(\ddot{x}+\left(\varepsilon A^{2}+\alpha\right) x-\left(\ddot{x}_{0}+\left(\varepsilon A^{2}+\alpha\right) x_{0}\right)\right)+p\left(\ddot{x}+\alpha x+\varepsilon x^{3}\right)=0
$$

Substituting (3.3a) and the initial conditions (3.2) into the homotopy (3.18) and equating the terms with identical powers of $P$, we obtain the following set of linear differ- 
ential equations:

$$
\begin{array}{ll}
p^{0}: \ddot{x}_{0}+\alpha x_{0}+\varepsilon A^{2} x_{0}=0, & x_{0}(0)=A, \dot{x}_{0}(0)=0 \\
p^{1}: \ddot{x}_{1}+\alpha x_{1}-\varepsilon A^{2} x_{0}+\varepsilon A^{2} x_{1}+\varepsilon x_{0}^{3}=0, & x_{1}(0)=0, \dot{x}_{1}(0)=0 \\
p^{2}: \ddot{x}_{2}+\alpha x_{2}-\varepsilon A^{2} x_{1}+\varepsilon A^{2} x_{2}+3 \varepsilon x_{0}^{2} x_{1}=0, & x_{2}(0)=0, \dot{x}_{2}(0)=0
\end{array}
$$

Consequently, solving the above equations, the first few components of the homotopy perturbation solution for (3.19) are derived as follows:

$$
\begin{aligned}
x_{0}= & A \cos \left(\sqrt{\varepsilon A^{2}+\alpha} t\right) \\
x_{1}= & \frac{1}{32\left(\varepsilon A^{2}+\alpha\right)^{\frac{3}{2}}}\left(4 t \varepsilon A^{2} \sin \left(\sqrt{\varepsilon A^{2}+\alpha} t\right)-\cos \left(\sqrt{\varepsilon A^{2}+\alpha} t\right) \sqrt{\varepsilon A^{2}+\alpha}\right. \\
& \left.+4 t \alpha \sin \left(\sqrt{\varepsilon A^{2}+\alpha} t\right)+\varepsilon A^{3} \cos \left(3 \sqrt{\varepsilon A^{2}+\alpha} t\right) \sqrt{\varepsilon A^{2} \alpha}\right)
\end{aligned}
$$

Finally, we have:

$$
x(t)=x_{0}(t)+x_{1}(t)+x_{2}(t)+\ldots
$$

\section{Result and discussion}

Example 1 Consider the governing equations of motion of free undamped Duffing's oscillator [27-31]:

$$
\ddot{x}+x+x^{3}=0, \quad x(0)=A, \quad \dot{x}(0)=0
$$

Has observed that although the series can be rapidly convergent in a very small region, it has very slow convergence rate in the wider region and the truncated series solution is an inaccurate solution in that region, which will greatly restrict the application area of the method [27]. The comparison between ADM and exact solution was shown in this [27] and they have shown that ADM is valid for small region.

The presented HPM solution is obtained from (3.20), considering just the two first $\operatorname{term}\left(x(t)=x_{0}(t)+x_{1}(t)\right)$ and with substituting $\varepsilon, \alpha$ as 1,1 respectively. The standard HPM solution is obtained from the (3.5) with the 5 term $(i=0, \ldots, 4)$ and also substituting $\varepsilon, \alpha$ as the same with previous one. Figure 1 compares the different solutions with numerical solution when $A=1.8$, the same with [27]. In this case, HPM and ADM are just valid for short region, but the new modification HPM solution with two terms exactly the same with the numerical solution for this strongly nonlinear problem.

Figure 2 shows the comparison between numerical solution, standard HPM and new modification HPM for different amount of $A$. When $A$ reads 1 (Fig. 2(a)), the 


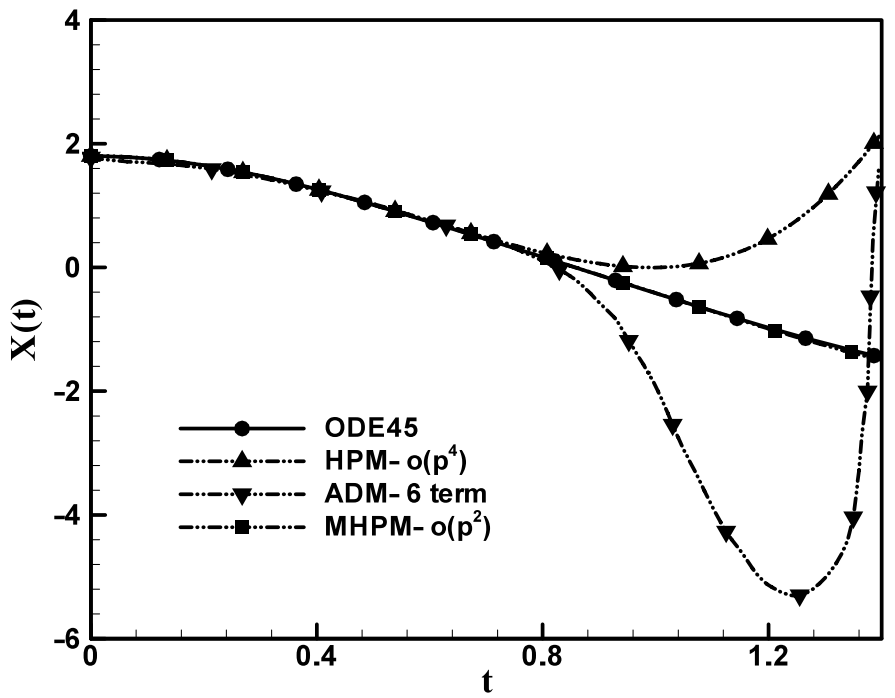

Fig. 1 The comparison between standard HPM, present HPM, ADM and numerical solutions for $A=1.8$ for Example 1

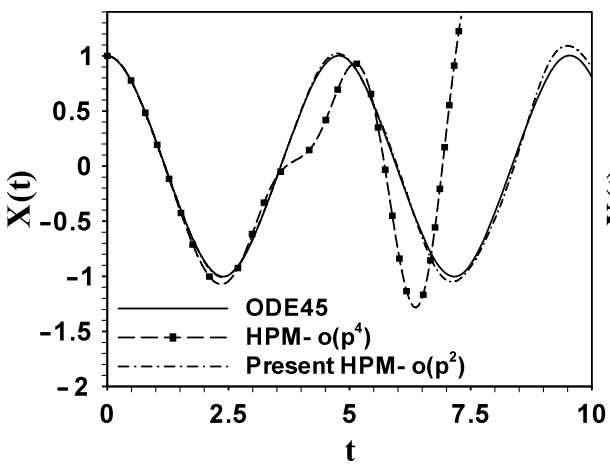

(a)

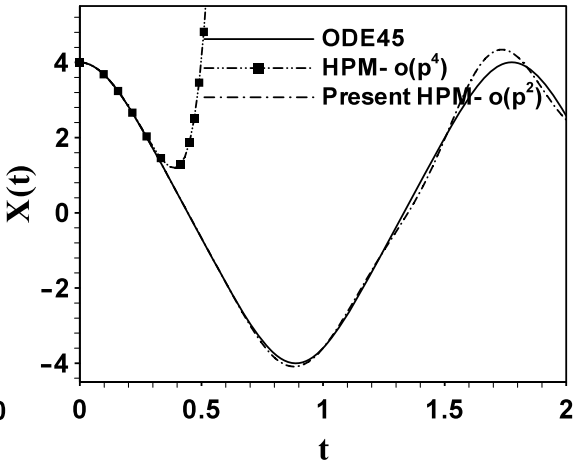

(b)

Fig. 2 The comparison between presented HPM, standard HPM and numerical solution for (a) $A=1$ and (b) $A=4$ for Example 1

new modification HPM solution is almost valid for large wide range of times $(t=10)$ that shows that present method can solve this strongly nonlinear oscillators with high degree of accurate by the two first terms only. When $A$ increases, our validity range is confined to smaller value of time. The result for $A=4$ have been shown in Fig. 2(b) that shows present solution validity range restricted to $t<4$, but we can increase the validity range with increasing the terms of solution to more than 2 terms. In both cases $(A=1,4)$, the standard HPM solutions with $O\left(p^{4}\right)$ are just valid for short region. 


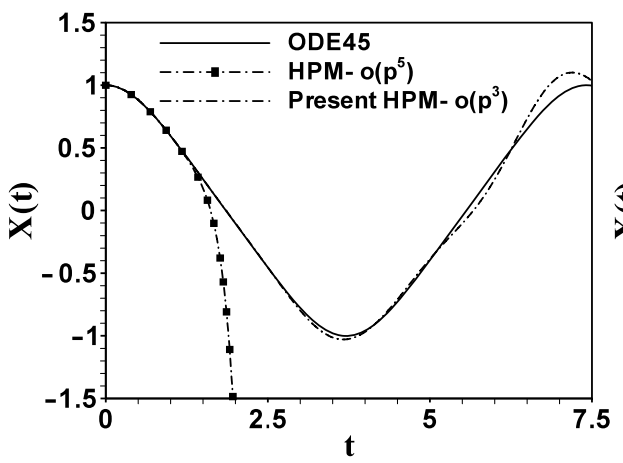

(a)

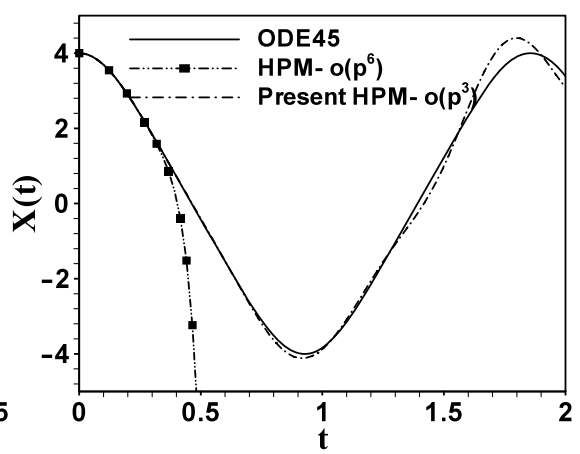

(b)

Fig. 3 The comparison between present HPM, standard HPM and numerical solution for (a) $A=1$ and (b) $A=4$ for Example 2

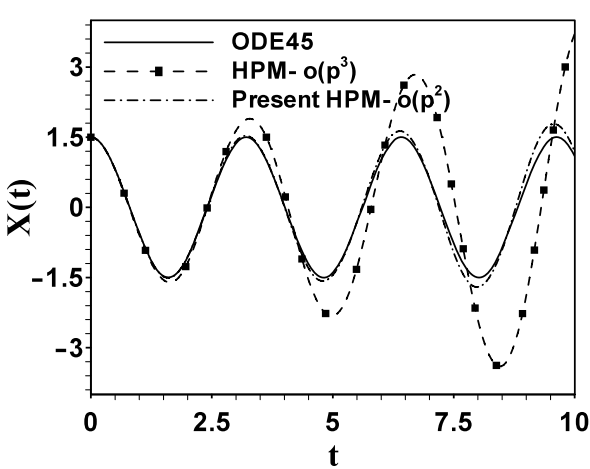

(a)

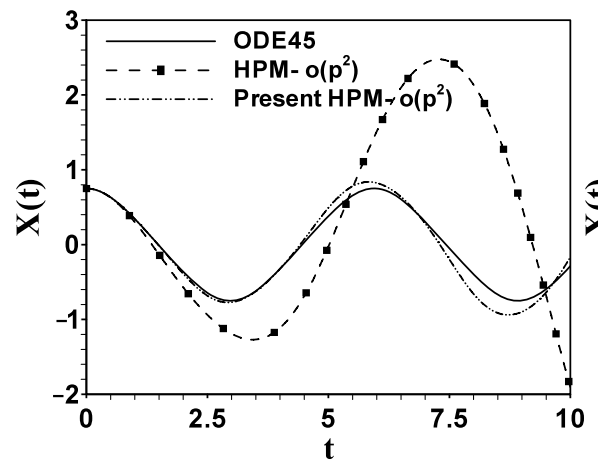

(c)

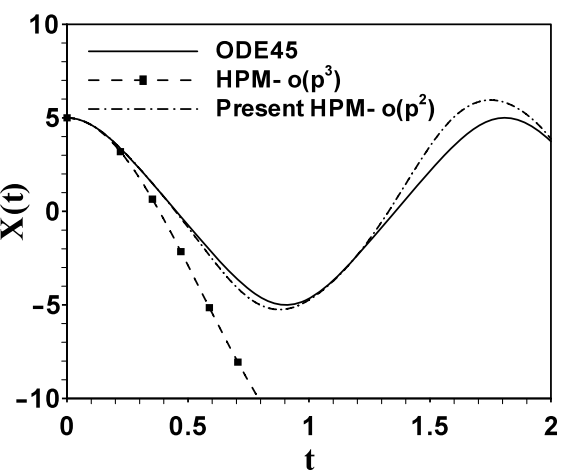

(b)

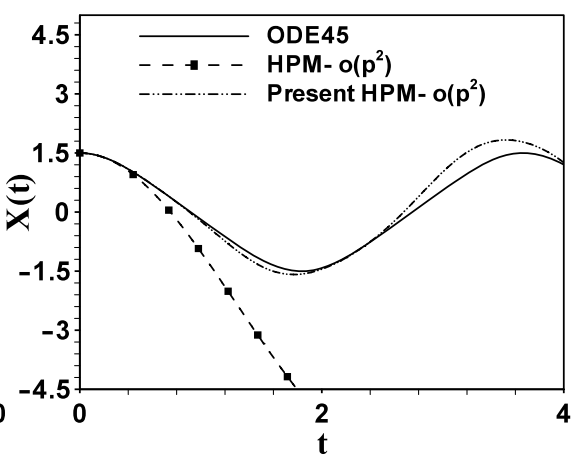

(d)

Fig. 4 The comparison between present HPM, standard HPM and numerical solution for (a) $A=1.5, \varepsilon=0.5, \alpha=3$, (b) $A=5, \varepsilon=0.5, \alpha=3$, (c) $A=0.75, \varepsilon=1.5, \alpha=0.5$ and (d) $A=1.5, \varepsilon=1.5, \alpha=0.5$ 
Example 2 Consider the governing equations of motion of oscillator as [28-30, 3235]:

$$
\ddot{x}+x^{3}=0, \quad x(0)=A, \quad \dot{x}(0)=0,
$$

Equation (3.20) gives us the new modification of HPM solution by substitution $\varepsilon, \alpha$ as 1, 0 respectively. In this case, the standard HPM solution is obtained with $\varepsilon=1$ and the (3.6) which specifies for $\alpha=0$. Figure 3 shows the comparison between numerical solution, standard HPM and new modification HPM for different amount of $A$. When $A$ reads 1 (Fig. 3(a)), the new modification HPM solution is almost valid for large wide range of times $(t=7.5)$. The result for $A=4$ have been shown in Fig. 3(b). In both cases $(A=1,4)$, the standard HPM solutions with $O\left(p^{6}\right)$ are just valid for short region.

Example 3 With substituting $\alpha=3$ and $\varepsilon=0.5$ in (3.1), we have:

$$
\ddot{x}+3 x+0.5 x^{3}=0, \quad x(0)=A, \quad \dot{x}(0)=0
$$

And with $\alpha=0.5$ and $\varepsilon=1.5,(3.1)$ is written as:

$$
\ddot{x}+0.5 x+1.5 x^{3}=0, \quad x(0)=A, \quad \dot{x}(0)=0
$$

Figure 3 shows the comparison between numerical solution, standard HPM and present HPM for different value of $A, \alpha$ and $\varepsilon$. These figures show validity of our new modification of HPM for different value of $\varepsilon$ and $\alpha$.

\section{Conclusion}

The new modified HPM is implemented to solve the $x^{3}$ force strongly nonlinear undamped oscillators and compared with numerical solution and standards HPM and ADM. The obtained results show that the new modification HPM is more accurate than the standard one and also this new method is valid for wide range. This method is more computationally efficient and it can give us high degree of accurate with two iterate.

Open Access This article is distributed under the terms of the Creative Commons Attribution Noncommercial License which permits any noncommercial use, distribution, and reproduction in any medium, provided the original author(s) and source are credited.

\section{References}

1. He, J.H.: Homotopy perturbation technique. Comput. Methods Appl. Mech. Eng. 178, 257-262 (1999)

2. He, J.H.: A coupling method of homotopy technique and perturbation technique for nonlinear problems. Int J. Non-Linear Mech. 35(1), 37-43 (2000)

3. Sadighi, A., Ganji, D.D.: Exact solutions of Laplace equation by homotopy-perturbation and Adomian decomposition methods. Phys. Lett. A 367, 83-87 (2007) 
4. Domairry, G., Ahangari, M., Jamshidi, M.: Exact and analytical solution for nonlinear dispersive $K(m, p)$ equations using homotopy perturbation method. Phys. Lett. A 368, 266-270 (2007)

5. Ganji, D.D., Rajabi, A.: Assessment of homotopy-perturbation and perturbation methods in heat radiation equations. Int. Commun. Heat Mass Transf. 33, 391-400 (2006)

6. Ganji, D.D., Afrouzi, G.A., Hosseinzadeh, H., Talarposhti, R.A.: Application of homotopyperturbation method to the second kind of nonlinear integral equations. Phys. Lett. A 371, 20-25 (2007)

7. Rajabi, A., Ganji, D.D., Taherian, H.: Application of homotopy perturbation method in nonlinear heat conduction and convection equations. Phys. Lett. A 360, 570-573 (2007)

8. He, J.H.: The homotopy perturbation method for nonlinear oscillators with discontinuities. Appl. Math. Comput. 151, 287-292 (2004)

9. Sadighi, A., Ganji, D.D.: Analytic treatment of linear and nonlinear Schrödinger equations: A study with homotopy-perturbation and Adomian decomposition methods. Phys. Lett. A (2007). doi:10.1016/j.physleta.2007.07.065

10. He, J.H.: Application of homotopy perturbation method to nonlinear wave equations. Chaos Solitons Fractals 26(3), 695-700 (2005)

11. Ganji, D.D., Nourollahi, M., Mohseni, E.: Application of He's methods to nonlinear chemistry problems. Comput. Math. Appl. 54, 1122-1132 (2007)

12. Ganji, D.D.: The application of He's homotopy perturbation method to nonlinear equations arising in heat transfer. Phys. Lett. A 355, 337-341 (2006)

13. Rafei, M., Ganji, D.D., Daniali, H.: Solution of the epidemic model by homotopy perturbation method. Appl. Math. Comput. 187, 1056-1062 (2007)

14. He, J.H.: Homotopy perturbation method: a new nonlinear analytic technique. Appl. Math. Comput. 135, 73-79 (2003)

15. He, J.H.: Comparison of homotopy perturbation method and homotopy analysis method. Appl. Math. Comput. 156, 527-539 (2004)

16. He, J.H.: Asymptotology by homotopy perturbation method. Appl. Math. Comput. 156, 591-596 (2004)

17. He, J.H.: Limit cycle and bifurcation of nonlinear problems. Chaos Solitons Fractals 26(3), 827-833 (2005)

18. He, J.H.: Some asymptotic methods for strongly nonlinear equations. Int. J. Mod. Phys. B 20(10), 1141-1199 (2006)

19. Cveticanin, L.: The homotopy-perturbation method applied for solving complex-valued differential equations with strong cubic nonlinearity. J. Sound Vib. 285, 1171-1179 (2005)

20. Odibat, Z.M.: A new modification of the homotopy perturbation method for linear and nonlinear operators. Appl. Math. Comput. 189, 746-753 (2007)

21. Beléndez, A., Pascual, C., Ortuño, M., Beléndez, T., Gallego, S.: Application of a modified He's homotopy perturbation method to obtain higher-order approximations to a nonlinear oscillator with discontinuities. Nonlinear Anal.: Real World Appl. (2007). doi:10.1016/j.nonrwa.2007.10.015

22. Beléndez, A., Pascual, C., Gallego, S., Ortuño, M., Neipp, C.: Application of a modified He's homotopy perturbation method to obtain higher-order approximations of an $x^{1 / 3}$ force nonlinear oscillator. Phys. Lett. A 371, 421-426 (2007)

23. Odibat, Z., Momani, S.: Modified homotopy perturbation method: Application to quadratic Riccati differential equation of fractional order. Chaos Solitons Fractals 36, 167-174 (2008)

24. Golbabai, A., Keramati, B.: Modified homotopy perturbation method for solving Fredholm integral equations. Chaos Solitons Fractals (2006). doi:10.1016/j.chaos.2006.10.037

25. Beléndez, A., Pascual, C., Beléndez, T., Hernández, A.: Solution for an anti-symmetric quadratic nonlinear oscillator by a modified He's homotopy perturbation method. Nonlinear Anal.: Real World Appl. (2007). doi:10.1016/j.nonrwa.2007.10.002

26. MATLAB ${ }^{\circledR} 6$ - The Language of Technical Computing (2006)

27. Ghosh, S., Roy, A., Roy, D.: An adaptation of Adomian decomposition for numeric-analytic integration of strongly nonlinear and chaotic oscillators. Comput. Methods Appl. Mech. Eng. 196, 11331153 (2007)

28. Ramos, J.I.: An artificial parameter-decomposition method for nonlinear oscillators: Applications to oscillators with odd nonlinearities. J. Sound Vib. 307, 312-329 (2007)

29. Ramos, J.I.: On the variational iteration method and other iterative techniques for nonlinear differential equations. Appl. Math. Comput. (2007). doi:10.1016/j.amc.2007.09.024

30. $\mathrm{Xu}, \mathrm{L}$.: Determination of limit cycle by He's parameter-expanding method for strongly nonlinear oscillators. J. Sound Vib. 302, 178-184 (2007) 
31. Hu, H., Tang, J.H.: A classical iteration procedure valid for certain strongly nonlinear oscillators. J. Sound Vib. 299, 397-402 (2007)

32. Beléndez, A., Beléndez, T., Márquez, A., Neipp, C.: Application of He's homotopy perturbation method to conservative truly nonlinear oscillators. Chaos Solitons Fractals (2006). doi:10.1016/j.chaos.2006.09.070

33. Lim, C.W., Lai, S.K.: Accurate higher-order analytical approximate solutions to non conservative nonlinear oscillators and application to van der Pol damped oscillators. Int. J. Mech. Sci. 48, 483-492 (2006)

34. He, J.H.: Variational iteration method-Some recent results and new interpretations. J. Comput. Appl. Math. 207, 3-17 (2007)

35. He, J.H., Wu, X.-H.: Variational iteration method: New development and applications. Comput. Math. Appl. 54, 881-894 (2007)

36. Xu, L.: Application of He's parameter-expansion method to an oscillation of a mass attached to a stretched elastic wire. Phys. Lett. A 368, 259-262 (2007)

37. Xu, L.: He's parameter-expanding methods for strongly nonlinear oscillators. J. Comput. Appl. Math. 207, 148-154 (2007)

38. Fo, Z., Mo, K., Demirbag, S.A.: Application of parameter-expansion method to nonlinear oscillators with discontinuities. Int. J. Nonlinear Sci. Numer. Simul. 9, 267-270 (2008)

39. Zhang, H.L.: Application of He's frequency-amplitude formulation to an $x(1 / 3)$ force nonlinear oscillator. Int. J. Nonlinear Sci. Numer. Simul. 9, 297-300 (2008)

40. He, J.H.: An elementary introduction to recently developed asymptotic methods and nano-mechanics in textile engineering. Int. J. Mod. Phys. B 22, 3487-3578 (2008)

41. He, J.H.: Recent development of the homotopy perturbation method. Topol. Methods Nonlinear Anal. 31, 205-209 (2008) 